\title{
The Discovery of Tidal Tails around the Globular Cluster NGC 7492 with Pan-STARRS1
}

\author{
C. Navarrete ${ }^{1,2,3}$, V. Belokurov ${ }^{3}$, and S. E. Koposov ${ }^{3,4}$ \\ ${ }^{1}$ Instituto de Astrofísica, Pontificia Universidad Católica de Chile, Av. Vicuña Mackenna 4860, 782-0436 Macul, Santiago, Chile; cnavarre@ astro.puc.cl \\ ${ }^{2}$ Millennium Institute of Astrophysics, Santiago, Chile \\ ${ }^{3}$ Institute of Astronomy, University of Cambridge, Madingley Road, Cambridge CB3 0HA, UK \\ ${ }^{4}$ McWilliams Center for Cosmology, Department of Physics, Carnegie Mellon University, 5000 Forbes Avenue, Pittsburgh, PA 15213, USA \\ Received 2017 April 17; revised 2017 May 10; accepted 2017 May 12; published 2017 May 25
}

\begin{abstract}
We report the discovery of tidal tails around the Galactic globular cluster NGC 7492, based on the Data Release 1 of the Pan-STARRS1 survey. The tails were detected using a version of the matched filter technique applied to the $(g-r, r)$ and $(g-i, i)$ color-magnitude diagrams. Tidal tails emerging from the cluster extend at least $\sim 3.5$ in the north-east to south-east direction, equivalent to $\sim 1.5 \mathrm{kpc}$ in projected length.
\end{abstract}

Key words: Galaxy: formation - Galaxy: halo - globular clusters: individual (NGC 7492)

\section{Introduction}

Globular clusters with tidal tails are precious for three reasons. First, there exists a coupling between the strength of the tides experienced by the cluster and its internal dynamics (see, e.g., Gnedin et al. 1999). Therefore, through simultaneous modeling of the bound and unbound stellar mass distributions, one can learn the details of the satellite's evolution in the Galaxy (see, e.g., Dehnen et al. 2004). Second, stellar tails grow approximately along the cluster's orbit (e.g., Eyre \& Binney 2011), thus providing a powerful technique to infer the properties of the host potential itself (e.g., Koposov et al. 2010; Bowden et al. 2015; Küpper et al. 2015). Third, globular cluster tails are fragile enough to be easily perturbed by low-mass objects, for example, dark matter subhalos with masses below $10^{8} M_{\odot}$ (e.g., Yoon et al. 2011; Erkal \& Belokurov 2015b). Thus, globular cluster streams are a unique tool for measuring the lumpiness of the Galactic dark matter distribution (Erkal \& Belokurov 2015a; Erkal et al. 2016).

A large number of the Milky Way globulars are predicted to be undergoing destruction today (see Gnedin \& Ostriker 1997); however, so far, tails have been detected only around a handful of objects (e.g., Odenkirchen et al. 2001; Belokurov et al. 2006; Lauchner et al. 2006; Niederste-Ostholt et al. 2010; Sollima et al. 2011). This discrepancy could perhaps be remedied by taking into account the effects of mass segregation: the masses of the stars shed by the cluster in the initial phases of the dissolution are simply too low to light up the tails (see Balbinot \& Gieles 2017). Additionally, this hypothesis may help to explain a substantial number of orphan stellar streams revealed so far (see, e.g., Grillmair \& Dionatos 2006b; Bonaca et al. 2012; Koposov et al. 2014; Balbinot et al. 2016). Most importantly, it serves to re-invigorate the search for tidal tails around globular clusters, albeit at lower surface brightness levels.

The absolute majority of the discoveries mentioned above have been made using the data from the Sloan Digital Sky Survey (SDSS), which, by now, has been trawled extensively for the Galactic stellar halo sub-structure. Motivated by the prospects of unearthing new examples of tidal disruption, we have searched for the presence of stellar tails around globular clusters located within the footprint of the Pan-STARRS1 (PS1) $3 \pi$ survey. Recently, after years of anticipation, the PS1 object catalogs were finally released publicly and are currently accessible for download through the MAST archive (see Flewelling et al. 2016). Compared to the SDSS, PS1 (i) provides continuous coverage at low Galactic latitudes in the northern hemisphere and (ii) extends $\sim 30^{\circ}$ further down under the celestial equator. Note that ours is not the first exploration of the Galactic stellar halo sub-structure with PS1 (see, e.g., Bernard et al. 2014, 2016).

In this Letter, we report the discovery of tidal tails around NGC 7492, a sparse outer halo Galactic globular cluster $\left(R_{\mathrm{GC}} \sim 25 \mathrm{kpc}\right.$; Harris 1996, 2010 edition; see also Figuera Jaimes et al. 2013). Previous studies of the cluster suggested the presence of extra-tidal stellar material. For example, Leon et al. (2000) found a small extension pointing towards the Galactic center, but emphasized the need for high-quality deep CCD data to confirm this low surface brightness structure unambiguously. Later, studying a field $42^{\prime} \times 42^{\prime}$ in size, Lee et al. (2004) noticed tail-like structures extending towards the northeast and northwest from the cluster. Given its mass and Galacto-centric distance, NGC 7492 was also tagged as a "tidally affected" cluster by Carballo-Bello et al. (2012). Other clusters included in this category are already known to have tidal tails, such as Pal 5 (Rockosi et al. 2002; Odenkirchen et al. 2003; Grillmair \& Dionatos 2006a), NGC 5466 (Belokurov et al. 2006), and NGC 5053 (Lauchner et al. 2006).

\section{PS1 Data}

The Panoramic Survey Telescope and Rapid Response System (Pan-STARRS, PS1) survey is observing the sky north of declination $-30^{\circ}$ with a $1.8 \mathrm{~m}$ optical telescope from the summit of Haleakala, in Hawaii. The telescope has a $7 \mathrm{deg}^{2}$ field of view, imaged with a mosaic CCD camera with 60 detectors, each with $4800 \times 4800$ pixels. The pixel scale is 0.258 arcsec. The images are obtained through five filters: $g P 1, r P 1, i P 1, z P 1$, and $y P 1$. The first data release (DR1) contains object catalogs produced using both non-stacked and stacked catalogs of the $3 \pi$ Steradian Survey. Details of the data delivered in PS1 DR1 are described in detail by Chambers et al. (2016) and Magnier et al. (2016a, 2016b). In the analysis presented here, stars were selected from the PS1 stack detection catalog considering only sources with (rpsfmagrkronmag) $<0.05$ (i.e., most likely stellar objects, see Farrow et al. 2014) and $g$ and $r$ STACK_PRIMARY flag-set greater than zero. The faintest magnitude considered was $r=22.5 \mathrm{mag}$. The 

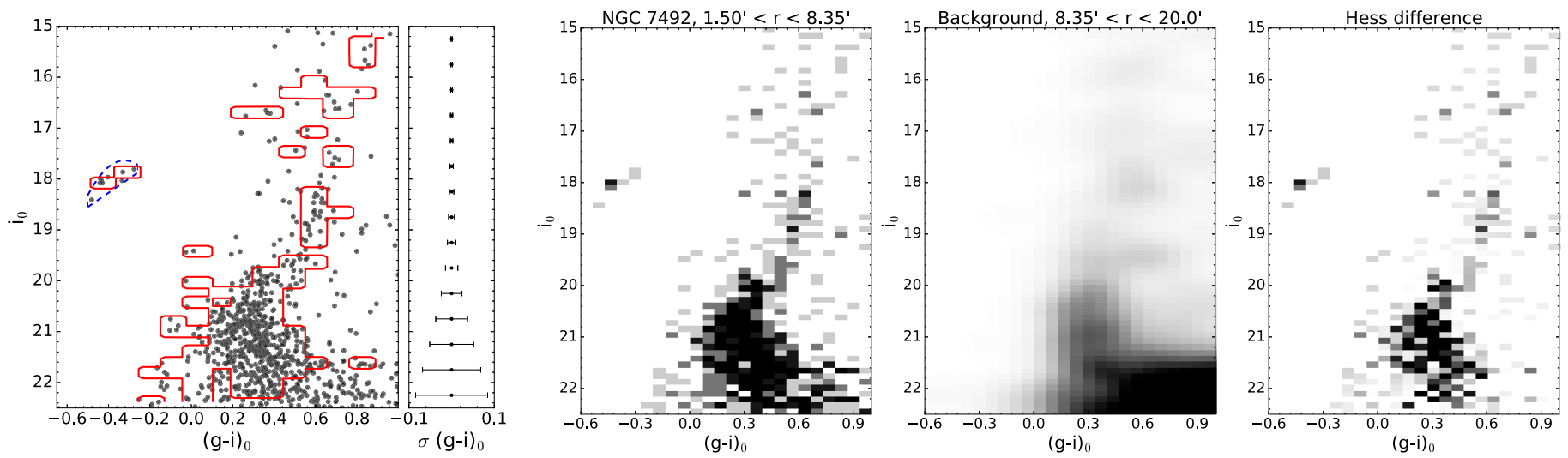

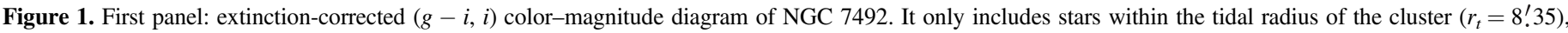

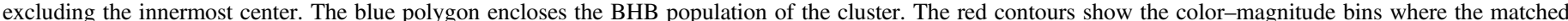

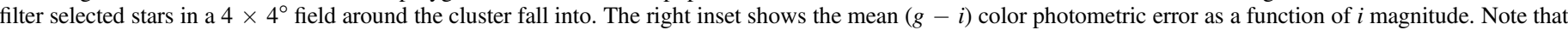

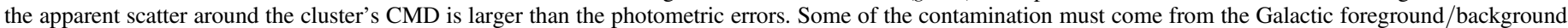

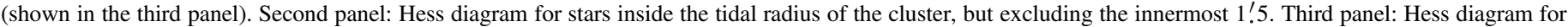

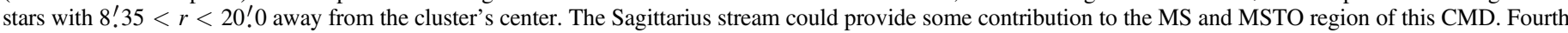

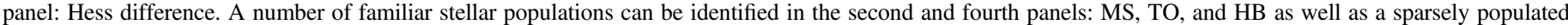
blue straggler sequence. In the background-subtracted Hess diagram, most of the contamination is removed, and the sequences are much narrower.

apparent magnitudes were corrected for extinction using the dust maps of Schlegel et al. (1998) as calibrated by Schlafly \& Finkbeiner (2011).

The left panel of Figure 1 shows the color-magnitude diagram (CMD) of the stars located inside the tidal radius of the cluster $\left(r_{t} \sim 8\right.$ !3, Harris 1996). To remove stars suffering from crowding, only objects outside the very center (i.e., $r>1$.5) are included. Despite contamination from field stars, the main sequence (MS), sub-giant branch, red giant branch (RGB), and blue horizontal branch (BHB) are clearly distinguishable. The CMD of the cluster also reveals the presence of a small number of stars brighter than the MS turn-off (TO), most probably blue straggler stars, as previously claimed by Cote et al. (1991). The RGB appears somewhat broadened: this scatter is difficult to explain given the current constraints on chemical abundance variations in the cluster, but very few giants from the cluster have spectroscopic studies (see Cohen \& Melendez 2005, and references therein).

The three rightmost panels of Figure 1 show the Hess diagrams (stellar density in color-magnitude space) for a field centered on the cluster (middle left panel), for an outer annulus (middle right), and their difference (right panel). The Hess diagram for the cluster field gives a clearer view of the satellite's stellar populations: an extended BHB at $-0.6<(g-i)<-0.3$ and $i \sim 18 \mathrm{mag}$, the MS populated mainly by stars fainter than $i=20 \mathrm{mag}$, and the TO point at $(g-i)=0.4$ and $i \sim 20 \mathrm{mag}$. While over-densities of stars around these evolution stages are clearly discernible, there is substantial contamination at the faintest and reddest magnitudes, as well as in the RGB region. The decontaminated Hess difference (right panel of the figure) allows one to peel away the foreground layer, thus making the cluster's stellar sequence much more evident. Here, reassuringly, the satellite's MS appears much tidier, and hints of the asymptotic-giant branch are even noticeable at magnitudes brighter than $i \sim 17$ mag.

Note that the cluster location on the sky at $(\alpha, \delta)=\left(347^{\circ} .1\right.$, $\left.-15^{\circ} .6\right)$ (J2000), or $l=53^{\circ} .38, b=-63^{\circ} .48$, NGC 7492 lies within the projected position of the Sagittarius (Sgr) trailing stream, as pointed out by Carballo-Bello et al. (2014). Curiously, among all Galactic globular clusters in the vicinity of the stream, it has the lowest probability to be associated with the dwarf according to the model of Law \& Majewski (2010). Nonetheless, we conjecture that a good fraction of the field contamination around NGC 7492 is supplied by the Sgr debris, given that both systems are at similar heliocentric distances and are located relatively close on the sky (see Figure 17 from Carballo-Bello et al. 2014).

\section{Detection of the Tidal Tails and Discussion}

To search for extra-tidal extensions to the cluster's light distribution, we broadly follow the matched filter methodology as described in Rockosi et al. (2002). Taking advantage of the PS1 multi-band photometry, we generate matched filter masks for both $(g-r, r)$ and $(g-i, i)$ CMDs. Only stars with $15.0<i, r<22.5$ were considered. These magnitude cuts avoid possible saturated stars and the faint stars with larger photometric errors. The color-magnitude distribution of the matched filter selected stars was constructed using the background-subtracted Hess diagrams for $(g-i, i)$ (rightmost panel of Figure 1) and the equivalent $(g-r, r)$ Hess subtracted diagram. For the foreground/background color-magnitude distribution, an adjacent area of the sky was used, excluding a window in right ascension around NGC 7492. The background CMD was constructed from four regions, defined at the boundaries: $339^{\circ}<$ R.A. $<342^{\circ}, 342^{\circ}<$ R.A. $<345^{\circ}$, $349^{\circ} .25<$ R.A. $<352^{\circ} .25, \quad 352^{\circ} .25<$ R.A. $<355^{\circ} .25$, and between $-20^{\circ}<$ Decl. $<-10^{\circ}$. The Hess diagrams of the four fields were averaged to obtain a mean background density of stars in the color-magnitude plane.

The ratio of the cluster's and the Galactic background's CMD densities (with pixel size $0.12 \times 0.2 \mathrm{mag}$ ) was used to define the selection region in the $(g-r, r)$ and $(g-i, i)$ planes independently. Any star can then be assigned a weight corresponding to the value of the Hess ratio of the CMD pixel it falls into. For instance, BHB stars tend to have higher weights, because the field color-magnitude distribution is less populated in that region. The weight assigned was the sum of the weights in the $(g-r, r)$ and $(g-i, i)$ planes. Using these two matched filters (or three photometric bands) simultaneously improves the signal of the stream significantly. It helps to reduce the impact of small fluctuations in the CMD of the 


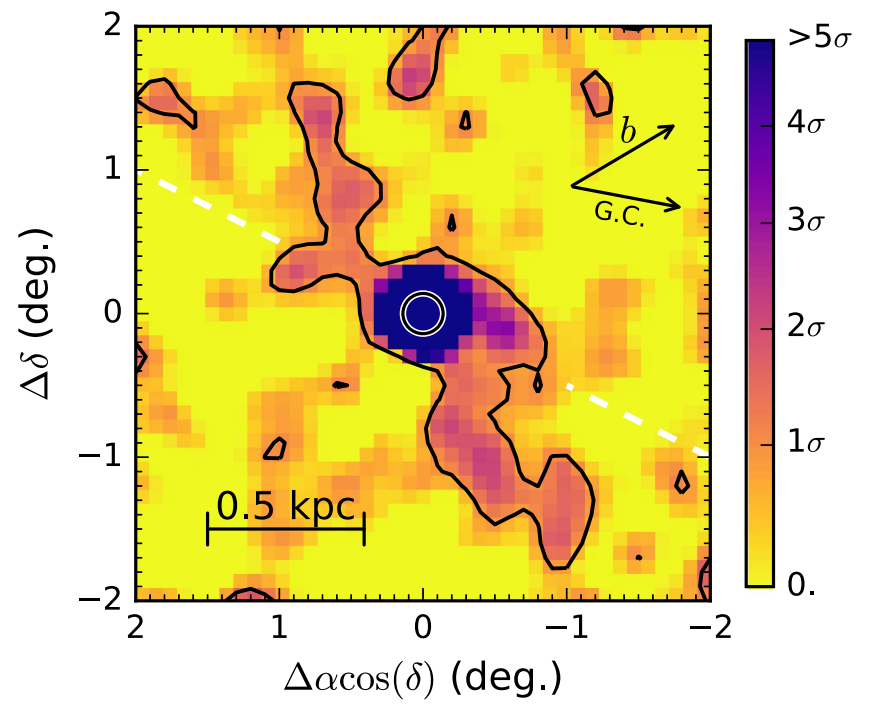

Figure 2. Logarithm of the density of stars that passed the two matched filters (see the main text). The map is $43 \times 43$ pixels across and was smoothed using a Gaussian filter with a standard deviation of 0.12 . The scale between degrees and the physical distance, in $\mathrm{kpc}$, is indicated by the bar at the left bottom corner. The innermost circle corresponds to the tidal radius of the cluster. The contour encloses the pixels with weights that are $>1 \sigma$ above the mean background. Note the two narrow stellar tails extending symmetrically from the cluster. As a reference, in the upper right corner, the direction towards the Galactic center (G.C.) and towards increasing Galactic latitude, $b$, values are indicated by the arrows. The dashed white line marks the direction of the Sgr stream stars according to the model from Law \& Majewski (2010).

cluster, which is sparsely populated to begin with, and additionally suffers from contamination from field stars even in the cluster innermost area (see Figure 1). Once the weight was assigned, only stars with weights above a certain threshold were considered as likely cluster members. The weight threshold is determined by maximizing the signal-to-noise of the cluster itself. This is the same approach as used by Erkal et al. (2016). The red contour in the left panel of Figure 1 shows the CMD bins where the matched filter selected stars from Figure 2 fall into. Despite some possible contamination from stars belonging to the Sgr stream, the stars selected seem to follow the MS and RGB of the cluster.

Figure 2 shows the density of the matched filter selected stars in the $4^{\circ} \times 4^{\circ}(1.8 \times 1.8 \mathrm{kpc})$ area centered on the cluster. The map was smoothed using a Gaussian filter with $\sigma=0$. 12 . The innermost circle marks the tidal radius of the cluster, and the contour line confines the pixels with values at $1 \sigma$ above the background. As evidenced from the figure, the matched filter reveals two tails on either side of the cluster, extending over $\sim 3.5$ ( $\sim 1.5 \mathrm{kpc}$ length) in the north-south direction. This structure resembles the characteristic "S-shaped" tidal feature found around other disrupting globular clusters, such as Pal 5 and NGC 5466. The contours drawn by Lee et al. (2004) are consistent with the northern branch and the small lobe found at $(\Delta \alpha \cos \delta$, $\Delta \delta) \sim\left(-0.7,-0{ }^{\circ} .1\right)$. Our results extend the northern branch on a factor of $\sim 5$ in length and also recover the southern branch, which was not seen previously. Given that there are no previous measurements of proper motions for this cluster, nor measurements in the Tycho-Gaia Astrometric Solution (TGAS Michalik et al. 2015; Lindegren et al. 2016) catalog, it is not possible to compare the direction of the stream with the projection of the orbit of the cluster, but in principle, both should be aligned. The white dashed line in Figure 2 marks the direction of the Sgr stream stars,
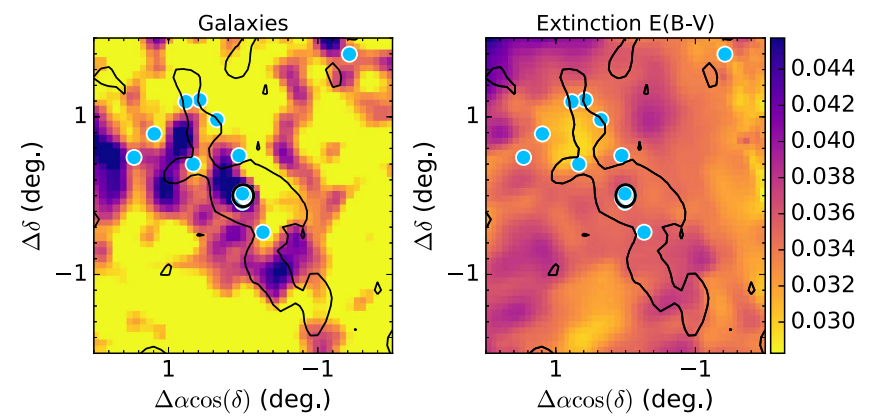

Figure 3. Left: number density of galaxies around the NGC 7492 globular cluster. The bin size and smoothing length are the same as those used in Figure 2. The innermost circle corresponds to the tidal radius, while the contour line corresponds to the pixels above $1 \sigma$ in the matched filter map of stars around the cluster (defined in Figure 2). Light blue filled circles give the positions of the BHB candidate stars at the distance of NGC 7492. Right: $E$ $(B-V)$ extinction around the cluster. The bin size and lines are the same as in the left panel. The color bar shows the mean extinction value per pixel.

according to the model of Law \& Majewski (2010). Despite the fact that the cluster is projected onto the expected location of the Sgr tidal stream, the dwarf's leading arm's direction is misaligned with the tidal tails detected in this work, thus making the possibility of an association of the two rather tenuous.

As a final check, we investigate whether some of the features seen in Figure 2 could be caused by misclassified galaxies and/ or effects of the interstellar extinction. The left panel of Figure 3 shows the logarithmic spatial distribution of galaxies (i.e., sources with (rpsfmag-rkronmag) $\geqslant 0.05$ ) around NGC 7492, selected based on the same matching filters as in the stellar density map and using the same bin size and smoothing kernel as in Figure 2. To guide the eye, the tidal radius of the cluster and the $1 \sigma$ contour of the matched filter density are over-plotted. There seems to be no direct correlation between the tails and the distribution of galaxies in the field. The variations in the extinction are unlikely to have affected our results, as indicated in the right panel of Figure 3. Clearly, the overall reddening of the Galaxy in the area is rather low (ranging between 0.03 and $0.045 \mathrm{mag}$ ), and its variation across the field does not correlate with tidal tails detected. Figure 3 also gives the positions of the BHB candidate stars, shown as light blue circles. These were selected to lie within the blue polygon in the left panel of Figure 1. Despite their overall low number density, BHBs appear to follow the northern tail, connecting its uppermost tip with NGC 7492 itself. On the other side of the cluster, however, there is a distinct lack of the BHB candidates. This perhaps is not surprising given the low number of BHB stars expected in a given stellar population. While the spatial distribution of the possible BHB candidates is suggestive, deeper wide imaging is required to confirm (and perhaps extend) the discovery presented here.

The Pan-STARRS1 Surveys (PS1) and the PS1 public science archive have been made possible through contributions by the Institute for Astronomy, the University of Hawaii, the PanSTARRS Project Office, the Max Planck Society and its participating institutes, the Max Planck Institute for Astronomy, Heidelberg and the Max Planck Institute for Extraterrestrial Physics, Garching, The Johns Hopkins University, Durham University, the University of Edinburgh, the Queen's University Belfast, the Harvard Smithsonian Center for Astrophysics, the Las Cumbres Observatory Global Telescope Network 
Incorporated, the National Central University of Taiwan, the Space Telescope Science Institute, the National Aeronautics and Space Administration under Grant No. NNX08AR22G issued through the Planetary Science Division of the NASA Science Mission Directorate, the National Science Foundation Grant No. AST-1238877, the University of Maryland, Eotvos Lorand University (ELTE), the Los Alamos National Laboratory, and the Gordon and Betty Moore Foundation.

The research leading to these results has received funding from the European Research Council under the European Union's Seventh Framework Programme (FP/2007-2013)/ERC Grant Agreement n. 308024. This project is supported by CONICYT's PCI program through grant DPI20140066. C.N. acknowledges support from CONICYT-PCHA grant Doctorado Nacional 201521151643. S.K. thanks Bernie Shiao for the assistance in retrieving the PS1 data. C.N. thanks Francisco Aros for his useful suggestion on the improvements of the figures.

\section{References}

Balbinot, E., \& Gieles, M. 2017, MNRAS, submitted (arXiv:1702.02543)

Balbinot, E., Yanny, B., Li, T. S., et al. 2016, ApJ, 820, 58

Belokurov, V., Evans, N. W., Irwin, M. J., Hewett, P. C., \& Wilkinson, M. I. 2006, ApJL, 637, L29

Bernard, E. J., Ferguson, A. M. N., Schlafly, E. F., et al. 2014, MNRAS, 443, L84

Bernard, E. J., Ferguson, A. M. N., Schlafly, E. F., et al. 2016, MNRAS, 463, 1759

Bonaca, A., Geha, M., \& Kallivayalil, N. 2012, ApJL, 760, L6

Bowden, A., Belokurov, V., \& Evans, N. W. 2015, MNRAS, 449, 1391

Carballo-Bello, J. A., Gieles, M., Sollima, A., et al. 2012, MNRAS, 419, 14

Carballo-Bello, J. A., Sollima, A., Martínez-Delgado, D., et al. 2014, MNRAS, 445, 2971

Chambers, K. C., Magnier, E. A., Metcalfe, N., et al. 2016, arXiv:1612.05560

Cohen, J. G., \& Melendez, J. 2005, AJ, 129, 1607

Cote, P., Richer, H. B., \& Fahlman, G. G. 1991, AJ, 102, 1358
Dehnen, W., Odenkirchen, M., Grebel, E. K., \& Rix, H.-W. 2004, AJ, 127,2753

Erkal, D., \& Belokurov, V. 2015a, MNRAS, 450, 1136

Erkal, D., \& Belokurov, V. 2015b, MNRAS, 454, 3542

Erkal, D., Koposov, S. E., \& Belokurov, V. 2016, MNRAS, submitted (arXiv:1609.01282)

Eyre, A., \& Binney, J. 2011, MNRAS, 413, 1852

Farrow, D. J., Cole, S., Metcalfe, N., et al. 2014, MNRAS, 437, 748

Figuera Jaimes, R., Arellano Ferro, A., Bramich, D. M., Giridhar, S., \& Kuppuswamy, K. 2013, A\&A, 556, A20

Flewelling, H. A., Magnier, E. A., Chambers, K. C., et al. 2016, arXiv:1612. 05243

Gnedin, O. Y., Lee, H. M., \& Ostriker, J. P. 1999, ApJ, 522, 935

Gnedin, O. Y., \& Ostriker, J. P. 1997, ApJ, 474, 223

Grillmair, C. J., \& Dionatos, O. 2006a, ApJL, 641, L37

Grillmair, C. J., \& Dionatos, O. 2006b, ApJL, 643, L17

Harris, W. E. 1996, AJ, 112, 1487

Koposov, S. E., Irwin, M., Belokurov, V., et al. 2014, MNRAS, 442, L85

Koposov, S. E., Rix, H.-W., \& Hogg, D. W. 2010, ApJ, 712, 260

Küpper, A. H. W., Balbinot, E., Bonaca, A., et al. 2015, ApJ, 803, 80

Lauchner, A., Powell, W. L., Jr., \& Wilhelm, R. 2006, ApJL, 651, L33

Law, D. R., \& Majewski, S. R. 2010, ApJ, 718, 1128

Lee, K. H., Lee, H. M., Fahlman, G. G., \& Sung, H. 2004, AJ, 128, 2838

Leon, S., Meylan, G., \& Combes, F. 2000, A\&A, 359, 907

Lindegren, L., Lammers, U., Bastian, U., et al. 2016, A\&A, 595, A4

Magnier, E. A., Chambers, K. C., Flewelling, H. A., et al. 2016a, arXiv:1612. 05240

Magnier, E. A., Sweeney, W. E., Chambers, K. C., et al. 2016b, arXiv:1612. 05244

Michalik, D., Lindegren, L., \& Hobbs, D. 2015, A\&A, 574, A115

Niederste-Ostholt, M., Belokurov, V., Evans, N. W., et al. 2010, MNRAS, 408, L66

Odenkirchen, M., Grebel, E. K., Dehnen, W., et al. 2003, AJ, 126, 2385

Odenkirchen, M., Grebel, E. K., Rockosi, C. M., et al. 2001, ApJL, 548, L165

Rockosi, C. M., Odenkirchen, M., Grebel, E. K., et al. 2002, AJ, 124, 349

Schlafly, E. F., \& Finkbeiner, D. P. 2011, ApJ, 737, 103

Schlegel, D. J., Finkbeiner, D. P., \& Davis, M. 1998, ApJ, 500, 525

Sollima, A., Martínez-Delgado, D., Valls-Gabaud, D., \& Peñarrubia, J. 2011, ApJ, 726, 47

Yoon, J. H., Johnston, K. V., \& Hogg, D. W. 2011, ApJ, 731, 58 